\title{
Liquid Separation: Three Fundamental Dimensions within LiquidFeedback and other Voting Technologies
}

\section{Nicolás Mendoza}

City University Hong Kong, nmendozal2-c@my.cityu.edu.hk

\begin{abstract}
The objective of this paper is to capitalize on the experiences of LiquidFeedback, providing insights that can be helpful for future developers of voting technologies. The condition of LiquidFeedback as a digital platform carries dramatic implications in terms of its ability to perceive, secure, and make effective the political expression of the community. Beyond the hype, can the LiquidFeedback platform accurately perceive the political will of participants? As a voting technology, is it socially located in a position from where it can meaningfully change the state of affairs of society? Is it configured so that it guarantees fairness and safety from both public and private perspectives? As a result of the conjunction of interviews and critical analysis, three fundamental dimensions to all voting technologies are proposed: Expressivity, Influence, and Integrity.
\end{abstract}

Keywords: digital democracy, voting technologies, Pirate Party, Liquid Democracy, LiquidFeedback

Acknowledgement: This research was conducted thanks to the support of the Centre for Digital Cultures (CDC) at Leuphana Inkubator, Leuphana University, Luneburg (Germany). Especial Thanks to Dr. Volker Grassmuck at CDC Leuphana Inkubator, and to Dr. Olli Tapio Leino at the School of Creative Media (SCM) at City University of Hong Kong.

\section{Introduction}

This research project aims to advance the understanding of voting technologies, through a series of in-depth interviews with individuals of diverse profile within the development of LiquidFeedback. The research, conducted in Germany during the summer of 2013, was structured in an open-ended way. Each interview generated new questions, gaps that needed to be filled, pointing the direction to be taken next. Often, through our conversations our interviewees would suggest us to talk about an emerging topic with another individual who had better knowledge of the topic in question. In this way the thread of this research took shape on its own, leading us from topic to topic, from person to person. Contacted subjects play diverse roles within the overall project, throwing light in 
different directions. This led us to talking with users, advocates, developers, and so forth. In terms of method, this open-ended approach, directed mainly by the dialogues and findings in the field, feedback from the process itself, as well as the way the material is read in the analysis, owes primarily to Actor-Network Theory (Latour 2007).

\subsection{Three Dimensions}

This paper is interested in liquid democracy as a voting technology, (i.e., as a set of artifacts and procedures involved in the voting practice). Voting technologies are meant to make explicit and intelligible the political will of the members of the society that deploys them. These collective decision-making practices are technological artifact-procedure assemblages in which one thing (the nuanced political stances and desires of individual humans) is transformed into another (discrete and decisive information in binary yes/no form, or at best as a selection from constrained and predetermined option pools). Voting technologies are thus media for the expression of the political will of participants. They are a communication vehicle for the transmission of messages from the private to the public sphere. The voting technology is a device through which private views and desires about the collective are encoded and translated into a public language that shows a course of action for the community. Expressivity emerges as the primary dimension in our theory of voting technologies.

However, throughout the series of interviews conducted during this process, at least two more dimensions fundamental to the nature of voting technologies emerged. Another dimension is what we call Influence, understood as the degree to which the tool is able to change the state of affairs within any given group. In other words, can the expressivity the tool affords actually influence the way things are in the community that uses it? Our last proposed dimension is that of Integrity, or the ability to meet both public and private expectations of security.

Notably, the dimension of Integrity contains within itself the struggle between the public and the private, and it is the realm of the tension between identity and anonymity in voting technologies. While the familiar technology of ballots, boxes, voting booths, ID cards, etc. deals with this tension mostly through physical means, the regime of material conditions specific to digital media implies a different set of procedural logics, often resulting in seemingly unresolvable contradictions between these two key aspects mentioned above: public integrity (identity) and private integrity (anonymity).

However, there are heuristics, minimum acceptable performance standards for these technologies. These are largely culturally determined. Due to the nature and function of voting, these basic criteria are necessarily always concerned with three dimensions: expressivity, integrity, and influence. The method should fulfil three requirements: it should indeed capture the will of the community, it should be trustable from both the perspective of the public and the state, and it should have the power to change things.

The three dimensions conform an analytical matrix, a space for the theory of all existing voting technologies. Our dimension-based analysis offers us the possibility to understand voting 
technologies in a way that is stripped from the fetishizing discourse of progress. In the continuum of the three dimensions, the pitfalls and virtues of diverse technologies from diverse times and places are evidenced, and electronics, boxes and hands get their fair praise and critique.

\subsection{All Liquids are not the same}

Liquid Democracy was not originally designed for use in traditional government. I didn't, and don't, want people to think of it as a quick-fix replacement for current governmental election methods. (...) You see, LD was originally designed for small, stealthy, distributed teams of anarchist kung-fu badasses. ${ }^{1}$

-John Washington Donoso (a.k.a Sayke), creator of Liquid Democracy

The topic of Liquid Democracy can be confusing because the catchword liquid has been attached to multiple ideas and projects, ideas and projects that nevertheless are fundamentally different between each other. "Liquid Democracy", an abstract concept, is often confused with "LiquidFeedback", the software package used by the Pirates in Germany. The original Liquid Democracy concept was first described sometime around the year 2000 (the exact date is hard to determine because numerous links and forum pages of the time are now inaccessible) by J.W. Donoso, a.k.a Sayke. For the sake of clarity I will refer to this, the original concept, from now on, with the term LD-Sayke.

Contrary to the general belief, LD-Sayke was not a software engine for proxy-voting decision making. LD-Sayke was the concept for a system that could, in theory, recommend better quality answers to the questions an online user might have. It proposed the idea of dynamic vote delegation, yes, but not as a mechanism to decide anything; merely as a method to sort out the order of the "answers" or results of a query. It must be stressed that the shift from "answer recommendation" to "decision-making" is a qualitative, non-trivial, one.

LD-Sayke was never meant to be a voting technology in the sense of, "a decision-making tool". LD-Sayke is actually a proposal for a way to organise content in function of its collectively perceived quality, a knowledge sorting system. If the term "decision-making system" has been associated with LD-Sayke, it is merely because better sorted knowledge allows the individual to make better decisions, but not because the system itself was ever meant to be used to output decisions. The key is that while better knowledge leads an individual to make better decisions, LDSayke was never intended as a system through which the collective would seal decisions:

"Vote proxying puts the power in the hands of the proxy - answer recommendation keeps the power in the hands of the people (or, at the edges of the network) where it belongs. (...) Vote proxying seems far too similar to representation in traditional democratic systems - rather then a mechanism through which we are informed by others, vote proxying (and traditional democratic representation) acts as a mechanism through which we cede power to others."

\footnotetext{
${ }^{1}$ http://seed.sourceforge.net/ld_k5_article_004.html
} 
These clarifications attempted by Sayke have been widely ignored. Current proponents work on a delegated-voting decision-making platform, while keeping the same "Liquid Democracy" name. This is how, in its website (https://liqd.net), the group Liquid Democracy e.V. defines "Liquid Democracy" in the following terms:

"The idea of "Liquid Democracy" has already been circulating on the web for a couple of years, however without any suggestions for a specific execution. The core of the idea is delegated voting, which removes the previous separation between representative democratic and direct democratic decisions. The principle of delegated voting is the base for all Liquid Democracy concepts." 2

Thus a new definition for "Liquid Democracy" emerged. This definition by the group Liquid Democracy e.V. departs in a fundamental way from the definition of LD-Sayke. Therefore I will subsequently refer to it with the term: $L D-e . V$.

Having clarified historical confusions, we are in the end left with a philosophical critique of LDe.V. (and by extension, of LiquidFeedback). Sayke argues that proxy-voting decision-making systems are more of the same because they still have representation at their core.

\section{The Voting Technologies Space}

\subsection{Expressivity}

Figure 1 shows a series of Brazilian citizens who participated in a social-media campaign titled \#NotEnough. They were asked to mark with a series of Xs the number of times they expected to participate in future elections:

Figure 1: A 4-year period electoral system "gives you 11 votes left to express everything you care about for the rest of your life!"3

2 www.liqd.net/en/schwerpunkte/theorische-grundlagen/liquid-democracy/

${ }^{3}$ http:/ / blog.parliamentofthings.org 


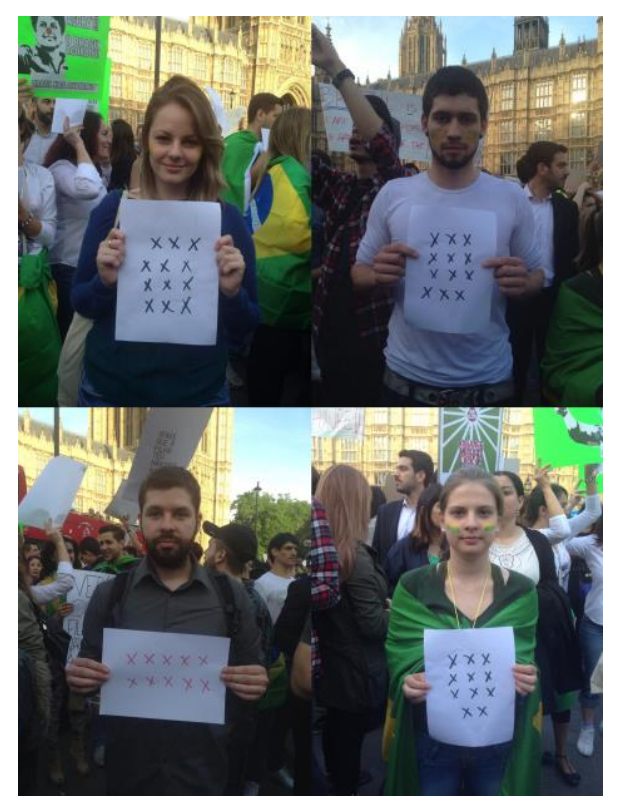

The \#NotEnough campaign stresses in a dramatic way the low expressivity of the currently predominant voting technology across the modern world.

However, expressivity in voting technology not only refers to frequency of vote occasions (time expressivity). It also refers to content expressivity. Is the system such that nuanced positions can be expressed, allowing to express opinions on diverse topics and issues? For example, if an individual believes there should be restrictions to fire arm ownership (in America, typically more of a Democrat-party position), and also believes reducing deficits should be a priority (which Republicans tend to support more), does the system by design possess the sensibility required for such nuance in opinion to be expressed, or must the voter sacrifice a part of their views?

There is also demographic expressivity. Can the technology perceive and encode the voice of the whole demographic universe of the population? Is it intelligible and accessible to all eligible voters, or only to a certain kind of person, and is it deployed in such a way that guarantees all voters can participate? Even ballot boxes are biased against the illiterate, disenfranchising them.

It can be argued that, out of the categories we just described, LD-e.V. and similar systems have no competition when it comes to the time expressivity and content expressivity categories. They leverage in clever ways the capabilities of digital networks to create what is perhaps the most expressive voting technology ever made. Indeed, participants here can express their views not periodically but permanently; choice is not broad and ideological, but nuanced, specific and practical; and, importantly, power transfer is not static but subject to change if for any reason a voter changes their mind.

While LD-e.V. excels in its ability to richly express the will of its users in nuanced and dynamic fashion, it fails dramatically in the requirement to express the demographic complexity of a large population. The price of personal computers puts them outside of the reach of an unacceptably large percentage of the population, and even the most affluent governments would struggle to 
provide sustained access to the required computational capabilities for every eligible voter. Moreover, computers themselves are epistemologically alien environments for too many people. Vast fractions of the population simply lack the relatively advanced digital literacies required to manage appropriately the complexity and transcendence of LD-e.V.

In the German town of Friesland, where an implementation of LD-e.V. called LiquidFriesland has been deployed, extremely low levels of participation are recorded. In June 2013, LiquidFriesland totalled only 300 registered users, most of them inactive. Djure Meinen, admin of LiquidFriesland described to us how low levels of engagement are actually perversely welcomed by politicians, who retort: "Democracy is an opportunity, and since now the opportunity is there, if people don't use it it is a problem of the people, not of the politicians." The argument effectively institutionalises political disenfranchisement. And, in this case, the existence of the LD-e.V. technology is actually used to legitimise the argument. The discourse of the voting technology appears emancipatory, yet its role in practice is to cement the status quo.

\subsection{Integrity}

The need for integrity is how a phenomenon like the voting ink comes to be: A gap in the credibility of the process is filled by incorporating a new gesture, the compulsion to submerge the finger in ink. The ink performs one of its material properties by attaching itself to the skin of the voter's finger, and another by changing the colour of the voter's skin. These two tricks afforded by the ink come together so that a third actor will notice the pigmentation alteration in case the owner of the finger intends to vote for a second time. Thus, a large scale version of this particular doublevoting attack becomes impractical enough, and so the ink restores reasonable credibility at the particular point of the process it is inserted in. The possibility of an ensemble of gestures and artifacts to provide trustable results is what we understand by the dimension of Integrity.

Voting technologies need to provide the community as a whole, and the participants as individuals, with guarantees that address the diverse vectors of attack that could compromise either the public or the private integrity, and the conflicting interests of different sectors of society. These guarantees must be embedded in the materiality itself of the technological assemblage of voting. Without guarantees a voting technology fails to set the foundation upon which trust in the process is rooted, and thus no lasting consensus can emerge around it. Low integrity renders a voting technology useless since the group is forced to seek alternative, trustable ways to achieve consensus.

Chapter 3 of the The Principles of LiquidFeedback book (Behrens et. al., 2014), “Open ballot vs. Secret Ballot", elaborates on how diverse voting technologies are in fact determined by equally diverse regimes of integrity. For the purpose of this report, let it suffice with the contrast presented in the book between the secret ballot and computer-based voting, as follows: 
Table 1: Secret Ballot / Computer-Based Voting Verifiability Comparison

\begin{tabular}{|l|l|}
\hline Verifiability of "secret voting with ballot box": & Verifiability of "voting computer type II": \\
\hline Only eligible voters cast a vote: limited verifiability & Only eligible voters cast a vote: not verifiable \\
\hline Only one vote cast by one voter: fully verifiable & Only one vote cast by one voter: not verifiable \\
\hline Votes are counted correctly: fully verifiable & Votes are counted correctly: fully verifiable \\
\hline
\end{tabular}

As we will see in the section dedicated to the topic of Influence, below, the verifiability limitations of this approach to computer voting results in serious limitations to the integrity, and thus to the ultimate potential influence of the technology.

The modern idea of society is such that secrecy and anonymity are non-negotiable characteristic of any voting technology that aspires to influencing public policy. Let it be clear, though, that this is not necessarily a universal imperative, just a modern one: Romain Bertrand notes in Towards a Historical Ethnography of Voting that one of the core and more deeply ingrained political assumptions of the West is that "there exists an indissoluble and common-sense link between some form of voting secrecy and individual political freedom" (Bertrand 2007, 1). At its core it is a fundamentally schizophrenic expectation countless societies have, for centuries, done perfectly without.

Integrity is a two way street: It should provide reasonable guarantees to the individual who uses it and it should also provide guarantees to the community. The individual should expect no personal negative consequences derived from using the voting technology. It must resist conceivable attacks to the privacy and overall safety of the voter. This is usually achieved through identity-obscuring mechanisms. We call this private integrity. Likewise, the community should be able to expect results that legitimately reflect the views of the community. The voting technology must resist conceivable attacks that attempt to unfairly distort the results themselves. Outsiders should not be able to vote, and insiders should be able to vote only once. This is usually achieved through identity-verification mechanisms. We call this public integrity.

Private integrity and public integrity are fundamentally at odds with each other. Private integrity works mainly by obscuring the identity and the interactions of the user, so that a malicious actor would not be able to determine who to target. Public integrity works mainly by determining and recording the identity and interactions of the user, so that legitimate actors are able to verify the process and determine whether the legitimacy of the process has been compromised at any point. Private integrity is based on anonymity, public integrity is based on verifiability.

This tension is one of the main reasons why voting needed to evolve as a technological affair in the first place. Devices and contraptions able to bridge the divide needed to be invented, and thus appeared the boxes, the booths, the ink, the ballots, and so forth. In these assemblages, each of 
these heterogeneous acts contributes its own specific affordances (the booth hides the voter, the ink marks him, etc.) so that the two conflicting integrities can be brought to a truce.

In the case of LiquidFeedback and the Pirates, Martin Haase describes the integrity-oriented measures in place, while highlighting that it is still flawed due to the meta-data trail problem:

Figure 2: The Three-Boxes Identity Clearing System

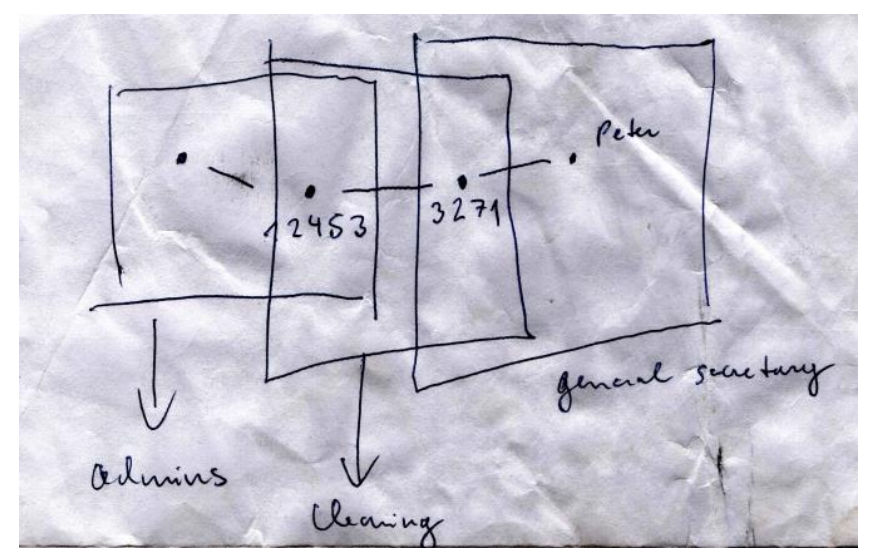

"The nickname is linked through a clearing point - an intermediary- so that a party functionary can not find out who is the person behind the nickname, this can only be found out through several people. (...) But we all know that of course this is relative anonymity, because in practice you can actually see what a certain nickname does, and if the nickname is active you can see how the nickname delegates to other people, you see who is linked to whom and identify people even if they don't reveal their identity by themselves" [anonymity is vulnerable to basic meta-data mining and meta-data analysis attacks. "one of the reasons why there is no strict anonymity is exactly this: because it is difficult in such a system where people interact to have anonymity, so anonymity is a false ideal because it can not be realised in real life"

As he speaks, Mr. Haase draws a series of overlapping boxes (Figure 2.) Later on, while listening again to the recording and contemplating his drawing, I cannot help but note how much Mr. Haase's boxes have in common with voting boxes and booths, the physical ballot boxes used to compartmentalize different parts and actors in regular secret ballot elections.

Another interviewee (Mr. Chan Hin) articulates the tensions of integrities in digital systems in the following simple formula:

"Anonymity, verifiability, digitality: pick two. The problem with anonymity in digital systems is that, you can make it anonymously and perhaps secure, but you have to have people who control it, administrators. So they can check that it's OK, so again you have a trust system. You have to trust the admins..." 


\subsection{Influence}

Systems like LiquidFeedback do not exist in a void. They are determined by their position relative to preexisting power structures as much as they are by their own internal design. LiquidFeedback as implemented by the German Pirate Party behaves in a fundamentally different way from LiquidFeedback as implemented by the local council of Friesland in their LiquidFriesland project, to the point that they should be considered different tools altogether. It is tempting to place our hopes for change and liberation from corporate rule to a tech tool, a compulsion for which Evgeny Morozov (Morozov 2013) has coined the term "technological solutionism".

The position of the voting technology relative to the society in which it is deployed determines its level of influence in the state of affairs. To what degree does the voting technology actually have an impact on the way things are, or will things ultimately keep going in the same general direction regardless of even an intense use of the technology? Influence is the result of a wide range of conditions that determine the actual nature of the tool.

Who specifically is deploying the tool? The particular nature of the implementing entity, its ideological orientation, its constellation of interests, its specific regime of affordances in the social context (i.e., its power) will ultimately determine what parts of the state of affairs will be exposed to the influence of the decision-making tool. Who deploys the tool, in other words, establishes the potential ceiling for the tool's influence. This "ceiling of influence" is the scope or range of topics that are admissible for debate. For instance, in the case of the Pirates, topics regarding the internal affairs of the Green Party would fall outside of what is admissible to debate, and thus outside the scope of the tool. However, entities with more power exist, like the German parliament, which are powerful enough to produce regulations that impact all political parties.

Interestingly, the actual limits of the voting technology influence are not solely determined by this "ceiling" or scope, which we observe to be ultimately theoretical. In practice it is often the deploying group itself who chooses to erode the potency of the tool beyond the theoretical limitations set by its ceiling. In the case of LD-e.V. type tools, we have seen that the actual potency of the tool tends to be diminished internally through diverse tactical and strategic considerations, severely reducing the meaningfulness of the whole exercise.

For instance, the position of the Pirate Party relative to general German society (and indeed of any political party) dictates that decisions made using their implementation of LiquidFeedback can only concern internal Party matters. This is the ceiling: Only Party matters, but potentially all Party matters. However, considerations regarding the integrity of the tool, especially the private integrity, as previously described, ultimately limit the influence of the tool. In practice it is reduced to being merely an aid to drafting the agenda for the Party's physical convention, where the meaningful decisions are taken by the method of the show of hands or the secret ballot.

The point here is to highlight the progressively declining influence of the tool given its location within social forces and other diverse factors that affect it: From the high-level expectation that the word "democracy" evokes, to the limited realm of the confines of the Party, to the even more limited realm of the Party's convention. Notably, time expressivity, one of the main "features" of the 
whole system -its ability to enable dynamic participation over time- is largely neutralised by this manoeuvre, given that the convention is an infrequent event.

Let us observe how the influence ceiling of the LiquidFriesland tool (from the northern town Friesland) is described by Djure Meinen, one of the system's admins:

"The topics are quite narrow. It's about schools, streets, financial questions. What do we have else? For example ... and yes, it's also streets and cars. (...) In Germany, we have, for example, with the streets, streets like the motorways that are owned by the federal state. Then, we have other roads that are owned by the state, and then we have roads that are owned by the District, and other roads that are owned by the towns (...) but the District can decide on their own roads, and so in Friesland, we only can discuss about ... a certain kind of road."

The ceiling, is determined by the fact that LiquidFriesland is an initiative of the local government. But the influence ceiling only determines the potential; invariably, the initiating entity exercises its power to impose further limitations:

"In the end, it's not a decision, but it's kind of making suggestions. The real decision is made by a council, but before it comes to the decision in the council, there are two possibilities for people using the Friesland to make suggestions to the council. First one is they can make a suggestion on their own, put it into LiquidFriesland, and then kind of discuss it and everyone who is in the system can decide on the suggestion, if it's a good suggestion or not. That's one thing. The other thing is the council can say we have a decision here we want to decide on in the next time, and put it into LiquidFriesland, and then people can decide if they think it's a good idea or not."

The "decision-making tool" is actually to make decisions about what to suggest to the actual decision-making body. In terms of influence, a disappointment. The initial influence ceiling of the tool is significantly higher, but the proponents of the tool -the local council- limit the real influence of the tool to the point where one is tempted to ask: How less influential could it be and still have a reason to even exist?

The initiating entity, whether it is a party, a council, or a grassroots organisation, will transfer its DNA to the voting technology. This means not only that this DNA will shape the topics and tone of debates, but more importantly, it will determine the power and practical influence resulting from the use of the technology. The tool is as powerful as its adopting entity, rather than the other way around. Hence,

Given meaningful differences in the context in which it is deployed, and in the position it occupies relative to existing power structures, the voting technology thus performs differently, ultimately becoming a different tool altogether.

The importance of this analytical concern is that focusing exclusively on the idea of Liquid Democracy (or, even digital democracy), often leads to the fetishisation of the decontextualized tool. The danger is to employ an excess of the vital political energy of the group on the matter of the vote itself, while forgetting about the real struggle for power, where the influence to change things comes from. Only if the technology and its analysis is considered as inextricable from 
context, the risk of tech fetishisation is contained, and we can move forward in understanding and advancing both: the technical and the political struggles, at the same time.

\subsubsection{Floating Devices}

In a previous section we have discussed how the term Liquid Democracy originated as an answerrecommendation system, and how later on it mutated to designate a different kind of system, a dynamic delegated decision-making platform. However the actual experiences of communities with systems of this kind (LD-e.V.) tends to evolve in the opposite direction. In short, perhaps one of the most interesting findings in the analysis of our interview material is as follows:

Communities that use L.D.-e.V. platforms tend to significantly reduce the influence of the platform. Indeed, in practice, the deploying communities themselves tend to retreat the tool from its decision-making purposes, back to the historical origins of the LD-Sayke platform as a questionanswering system.

In our interviews with LiquidFriesland and Pirate Party insiders there always came a moment where, not without a certain subtle embarrassment, our interviewees would reveal that in fact no decisions whatsoever were actually ever taken as a result of the use of the platform! As developer Christophe Chan Hin explained:

"We can't make decisions with LiquidFeedback in our party because we have this conflict [regarding privacy] and people said 'OK we use it with pseudonyms', but that isn't really accountable, so we can't use it for real decisions, so we only use it actually for setting the agenda. Basically that's it. (...) the reason why we don't make decisions in the party [using LiquidFeedback], is because its impossible to stay anonymous. We don't decide political issues, but we use the system to discuss them, actually."

But, if the system is not used to make binding decisions, what exactly is it used for?

"It is used for polling: you have an idea, and if many people say it is a good idea, then it has a social effect, you say $90 \%$ likes this proposal, so we have to do it in the next assembly."

So, LiquidFeedback is used neither for decision-making not for debate, but for 'polling', or more accurately for floating ideas. Martin Haase further explains: "The Party Congress didn't want to give up any privileges, the Party Congress wants to decide in the end..."

What we observe, then, is a fascinating inversion between how the proponents of the platform portray its purpose and the way it is actually used. The platform presented as a decision-making system that has no room for debate is actually used for debate, "proposal development", the floating of projects of ideas. Indeed LiquidFeedback is presented as "a powerful and so far unrivalled proposition development and decision-making software." (Behrens et. al. 2014, 127) Yet in practice, it is surprisingly power-less. Influence is eroded by the communities themselves upon realising its diverse shortcomings on the one hand, and by the incumbent powers who, come the moment of truth, are reluctant to yield their power to the stream of liquid delegation. 


\subsubsection{Protocols, the Liquid Wars and the Crafting of Voting Hybrids}

Finding ways to use digital networks to conduct highly expressive and legitimate democratic processes that can actually influence things is a holy grail of sorts. Observation of LiquidFeedback has made visible the three dimensions of voting technologies.

Looking forward, it is important for theorists, proponents and developers of future decisionmaking technologies to maintain awareness of these dimensions (Expressivity, Integrity, and Influence) to maximise the effectivity of their efforts and passion. Here we have only scratched the surface towards understanding the subtleties and paradoxes of these dimensions.

One of the biggest barriers LD-e.V. faces concern the problem of information asymmetry. At its root the problem of the "untrustable administrator" is inherent to the core/front-end architecture. Because of the core/front-end paradigm, a wide range of vital processes, like identity management, are centralised creating the unsolvable dilemmas concerning private integrity we have observed. The core/front-end architecture infuses system administrators with unacceptable informational privileges, and thus limits the possibilities for adoption and success of the whole platform.

A solution to this conundrum, which raises serious concerns in the Integrity dimension, might lie in structuring the entirety of the decision-making platform on a decentralised architecture, but that requires starting from scratch: Re-thinking the whole system from the basis of the fullydecentralised paradigm. There are currently several projects, like DemocracyOS http://democracyos.org/, that look into the potential of blockchain technology (the decentralised public ledger architecture underlying the Bitcoin protocol) to power the next generation of voting technologies. A fundamentally different decentralised architecture eliminates the need to trust system admins. The model here is to shift from a platform to a protocol, so it becomes necessary to start thinking with the grammar of protocols (see Figure 3.)

It remains to be seen to what extent these architectures imply decentralisation of power itself as much as decentralisation of procedure and instruments. Do technologies of radical decentralisation imply the dawn of the politics of decentralised power, and if so, what do these look like and how should they be constructed? 

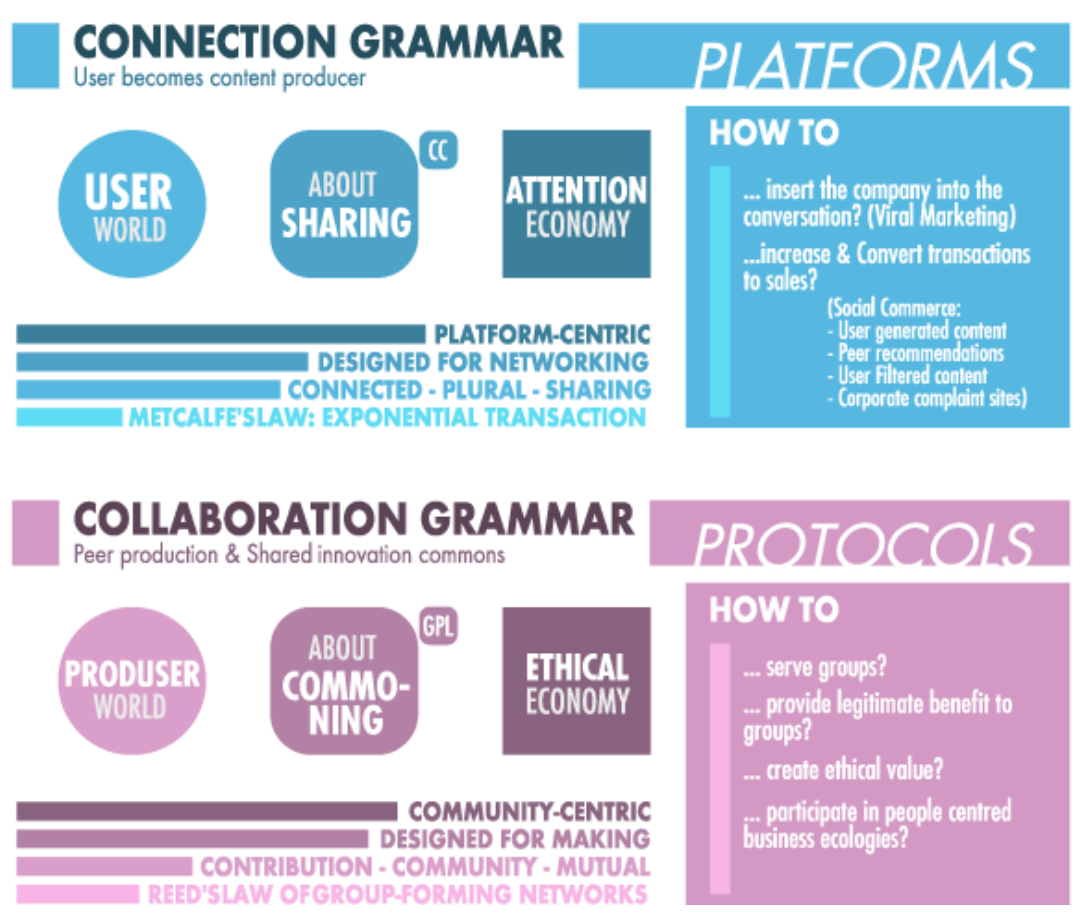

Figure 3: "The Relational Grammar of Internet Communication and Cooperation" (fragment)

In our conversation with Mr. Chan Hin, he succinctly diagnosed: "The solutions have to be combinations, I'm sure that this is the only way to do it." Indeed, in addition to the preceding suggestions (to focus on the three dimensions, and to consider the shift towards a grammar of protocols), we shall add a final one: to explore hybridisation. Indeed, the complexity of man and of the world -and especially of the modern world- practically guarantees that no "pure" approach can provide solutions for the needs of diverse nature and conflicting expectations that are demanded from voting technologies.

\subsubsection{UPDATE September 2015}

In a press release ${ }^{4}$ that elaborates on the major shortcomings of the LiquidFeedback platform, the Party acknowledged its use led to the internal conflict described above as the Liquid Wars: "the mother of all battles among factions". The structural flaws in LiquidFeedback thus led to its ultimate demise. Shortly after the completion of this article, in May 2015, the LiquidFeedback software instance used by the German Pirate Party was finally taken offline ${ }^{5}$.

\footnotetext{
${ }^{4}$ https:/ / www.piratenpartei.de/2015/06/07/die-mutter-aller-fluegelkaempfe-der-kampf-um-die-macht-inder-demokratie-2-0/

${ }^{5}$ https://vorstand.piratenpartei.de/2015/05/18/teamleiter-projektgruppe-relaunch-liquidfeedback/
} 
The reflective nature to the closing words of this press release, an official epitaph to the Pirates' adventure on the LiquidFeedback seas, echoes our final sentiment. Even if this specific implementation of the concept might have been irreparably flawed, the experience of the Pirates with it is of value in the search for better democratic arrangements:

"However, the debate on new forms of democracy is still far from over. (...) there are many experiences, evaluations [to be conducted] and improvements [to be implemented] until we can really say at some point that we have arrived at democracy 2.0. -"Street Dog", Pirate. ${ }^{6}$

\section{References}

Bauwens et. al. (2012) Synthetic overview of the collaborative economy. Technical report, P2P Foundation, Thailand, France, UK

Behrens et. al. The Principles of LiquidFeedback. Interaktive Demokratie e. V., Berlin, 2014.

R. Bertrand, et. al. (2007) Cultures of Voting: The Hidden History of the Secret Ballot Foundation nationale des sciences politiques. Centre d'études et de recherches internationales.

Latour, B. (2007) Reassembling the Social: An Introduction to Actor-Network Theory. Oxford University Press, USA

Morozov. E. (2013) To Save Everything, Click Here: The Folly of Technological Solutionism.internationals PublicAairs

\section{About the Author}

Nicolás Mendoza Leal is a PhD candidate at School of Creative Media, City University of Hong Kong.

${ }^{6}$ (Ibid. Footnote 4) 